\title{
Early Versus Late Recurrence of Hepatocellular Carcinoma After Surgical Resection Based on Post-recurrence Survival: an International Multi-institutional Analysis
}

\author{
Tao Wei $^{1,2} \cdot$ Xu-Feng Zhang ${ }^{1} \cdot$ Fabio Bagante ${ }^{3,4} \cdot$ Francesca Ratti $^{5} \cdot$ Hugo P. Marques $^{6} \cdot$ Silvia Silva $^{6}$. \\ Olivier Soubrane ${ }^{7}$. Vincent Lam ${ }^{8} \cdot$ George A. Poultsides ${ }^{9} \cdot$ Irinel Popescu ${ }^{10} \cdot$ Razvan Grigorie $^{10}$. \\ Sorin Alexandrescu ${ }^{10}$. Guillaume Martel ${ }^{11}$. Aklile Workneh ${ }^{11}$. Alfredo Guglielmi ${ }^{4} \cdot$ Tom Hugh $^{12} \cdot$ Yi Lv $^{1}$. \\ Luca Aldrighetti ${ }^{5}$. Timothy M. Pawlik ${ }^{3}$ (D)
}

Received: 1 January 2020 / Accepted: 18 February 2020 / Published online: 3 March 2020

(C) 2020 The Society for Surgery of the Alimentary Tract

\begin{abstract}
Background To define early versus late recurrence based on post-recurrence survival (PRS) among patients undergoing curative resection for hepatocellular carcinoma (HCC).

Methods Patients who underwent curative-intent resection for HCC between 2000 and 2017 were identified from an international multi-institutional database. The optimal cut-off time point to discriminate early versus late recurrence was determined relative to PRS.

Results Among 1004 patients, 443 (44.1\%) patients experienced recurrence with a median recurrence-free survival time of 12 months. A cut-off time point of 8 months was defined as the optimal threshold based on sensitivity analyses relative to PRS for early $(n=165,37.2 \%)$ versus late relapse $(n=278,62.8 \%)(p=0.008)$. Early recurrence was associated with worse PRS (median PRS, 27.0 vs. 43.0 months, $p=0.019$ ), as well as overall survival (OS) (median OS, 32.0 versus 74.0 months, $p<0.001$ ) versus late recurrence. In addition, patients who recurred early were more likely to recur at extra- \pm intrahepatic $(35.5 \%$ vs. $19.8 \%, p=0.003)$ sites and were less likely to have the recurrence treated with curative intent $(33.8 \%$ vs. $45.7 \%, p=0.08)$. Patients undergoing curative re-treatment of late recurrence had a comparable OS with patients who had no recurrence (median OS, 139.0 vs. 140.0 months); patients with early recurrence had inferior OS after curative re-treatment versus patients with no recurrence (median OS, 69.0 vs. 140.0 months, $p=0.036$ ), yet still better than patients who received palliative treatment for early recurrence (median OS, 69.0 vs. 21.0 months, $p<0.001$ ).

Conclusions Eight months was identified as the cut-off value to differentiate early versus late recurrence. Curative-intent treatment for recurrent intrahepatic tumors was associated with reasonable long-term outcomes.
\end{abstract}

Keywords Hepatocellular carcinoma $\cdot$ Resection $\cdot$ Early recurrence $\cdot$ Late recurrence $\cdot$ Post-recurrence survival

\section{Introduction}

Hepatocellular carcinoma (HCC) is a lethal malignancy with a 5-year survival of 20-40\%. [ $\left.{ }^{1}\right]$ Surgical resection remains the

Tao Wei and Xu-Feng Zhang contributed equally to this work.

Electronic supplementary material The online version of this article (https://doi.org/10.1007/s11605-020-04553-2) contains supplementary material, which is available to authorized users.

Timothy M. Pawlik

Tim.Pawlik@osumc.edu

Extended author information available on the last page of the article mainstay curative treatment option for early stage HCC. Long-term outcomes after curative resection of HCC are generally poor; however, due to a high incidence of recurrence, $\left[^{2}\right.$, ${ }^{3}$ ] understanding the timing and patterns of recurrence is therefore important. In particular, timing of recurrence has a demonstrated impact on prognosis, as early recurrence has been associated with inferior clinical outcomes among patients undergoing curative resection for HCC. $\left[{ }^{4}\right]$ In particular, early versus late recurrence has generally been classified using 2 years as the cut-off time point based on the assumed underlying mechanisms of relapse. [ ${ }^{5,6}$ ] Specifically, early recurrence within 2 years of surgery has generally been considered to be recurrence of intrahepatic micro-metastasis, whereas late 
recurrence has been suggested to represent de novo tumor due to multi-centric tumorigenesis. $\left[{ }^{7}\right]$ This paradigm of recurrence has not been validated, and emerging genomic evidence has suggested a more nuanced situation. For example, a recent study by Ding and colleagues demonstrated that $33.3 \%$ of HCC relapses within 2 years after resection were indeed multi-centric HCC arising from an independent tumor lineage compared with the initial tumor; in contrast, $36.4 \%$ of late recurrent tumors appeared to derive from disseminated tumor cells from the removed primary HCC. $\left[{ }^{8}\right]$ Several other studies have suggested that earlier relapse was associated with worse prognosis after recurrence in HCC. $\left[{ }^{9,}{ }^{10}\right]$ From a clinical standpoint relative to patient management, identifying patients susceptible to early recurrence is important as these patients may benefit from more intensive surveillance after surgery.

Post-recurrence survival (PRS) has been adopted as an indicator to determine the optimal cut-off time point to discriminate early versus late recurrence among patients undergoing curative resection for pancreatic ductal adenocarcinoma. $\left[{ }^{11}\right]$ Up to now, a clear evidence-based definition of early versus late recurrence has been lacking for patients undergoing resection of HCC. Therefore, the objective of the current study was to establish a cut-off value to differentiate between early versus late relapse relative to PRS, as well as identify potential risk factors associated with early recurrence.

\section{Methods}

\section{Study Cohort}

Patients who underwent curative-intent resection for HCC between 2000 and 2018 were identified from an international multi-institutional database. Patients were treated at 1 of the following 11 institutions: The Ohio State University Wexner Medical Center, Columbus, OH, USA; The First Affiliated Hospital of Xi'an Jiaotong University, Xi'an, China; University of Verona, Verona, Italy; Hopedale San Raffaele, Milano, Italy; Curry Cabral Hospital, Lisbon, Portugal; APHP, Beaumont Hospital, Clichy, France; Westhead Hospital, Sydney, Australia; Stanford University, Stanford, CA, USA; Funding Clinical Institute, Bucharest, Romania; University of Ottawa, Ottawa, Canada; The University of Sydney, School of Medicine, Sydney, Australia. The study was approved by the Institutional Review Boards of each participating institutions. All included patients were treatment-naïve before surgical resection and no patient received adjuvant therapy. Patients who died within 30 days after surgery were excluded $(n=25,2.4 \%)$.

\section{Clinicopathological Variables}

A standard datasheet was utilized to collect data at each institution. Demographic factors, including age, gender, status of alcohol intake, diabetes mellitus, and hepatitis virus infection, were collected from electronic records. Tumor-related characteristics, including maximum tumor size, tumor number and location, differentiation grade, presence of cirrhosis and microvascular invasion, underlying liver disease, liver capsule involvement, and width of resection margin, were collected based on final pathology. Liver function was classified using the Child-Pugh classification, whereas tumor burden was defined according to Barcelona Clinic Liver Cancer (BCLC) staging. Surgical information, including surgical approach (open versus laparoscopic), extent (major versus minor), and type (anatomic versus non-anatomic) of resection, were collected from the operation notes. Major hepatic resection referred to removal of three or more Cournand segments, while anatomic hepatic resection was defined by the Brisbane 2000 nomenclature of liver anatomy. Adjuvant therapies were not routinely adopted.

Primary outcomes were overall survival (OS) and recurrence-free survival (RFS), both calculated from the date of surgery. Recurrence was defined as identification of suspicious imaging findings or biopsy-proven tumor. Recurrence patterns including timing of recurrence, recurrence site (intrahepatic, extrahepatic, or both), and tumor number and size of recurrence as well as treatment approach for recurrent lesions were recorded. Local recurrence referred to relapse close to the resection margin of the liver tumor. Curative treatment options for relapsed HCC included surgical resection and ablation, while non-curative or palliative treatment included intra-arterial therapies, systemic chemotherapies, and targeted therapies, such as sorafenib. The adoption of curative versus palliative treatment was largely based on the BCLC stage of recurrent HCC. The secondary outcome was postrecurrence survival (PRS). Postoperative morbidity was also collected and graded as I-V according to the Clavien-Dindo classification. $\left[{ }^{12}\right]$

\section{Statistical Analysis}

Clinicopathological variables were summarized using frequencies plus percentages for categorical variables, while medians and interquartile range (IQR) were used for continuous covariates. Categorical covariates were compared with Chisquare test or Fisher's exact test and continuous variables with Mann-Whitney $U$ test. The OS and PRS were calculated using the Kaplan-Meier method and differences were compared using the log-rank test. Factors with a $P$ value less than 0.1 on univariate analysis were subsequently included in multivariable Cox regression model to identify independent risk factors associated with PRS. Similarly, associations between potential risk factors and early or late recurrence were assessed by univariate logistic regression analysis. Variables with $P$ values less than 0.1 were selected as covariate to enter multivariable logistic regression model. All statistical analyses 
were performed using SPSS version 23.0 (IBM SPSS, Chicago, IL, USA). A two-tailed $P$ value of $<0.05$ was considered statistically significant.

\section{Results}

\section{Study Cohort}

Among 1004 patients who underwent curative-intent liver resection for HCC, median age was 62 years (IQR, 53-71) and the majority $(n=789,78.6 \%)$ of patients were male. Most individuals presented with Child-Pugh grade liver function $(n=716,93.8 \%)$ and had BCLC stage 0/A tumors $(n=800$, $84.9 \%)$. Roughly, one-third $(n=291,30.1 \%)$ of patients underwent major resection; the majority $(n=799,82.6 \%)$ had an anatomical resection. An R0 margin was achieved in the overwhelming majority of patients $(n=902,90.0 \%)$ (Table 1). On final pathology, microvascular invasion was present in $32.5 \%(n=284)$ of HCC tumors. A total of 411 (41.4\%) patients experienced at least one complication after surgery; a small subset (8.8\%) of patients had severe (ClavienDindo III-IV) complications.

\section{Timing of Recurrence}

With a median follow-up of 28.0 months (IQR 14.0-47.0), $443(44.1 \%)$ patients experienced a recurrence following resection of the primary HCC. Cumulative recurrence at 1, 3, and 5 years was $21.9 \%, 46.6 \%$, and $58.0 \%$, respectively (Fig. 1). Perhaps not surprisingly, patients who developed recurrence were more likely to have presented with advanced tumor characteristics, such as large, multiple, and bilobar tumors, poor differentiation, and microvascular invasion (all $p<0.05$, Table 1). Among the 443 individuals who developed recurrence, the median time from initial surgery to recurrence was 12.0 (IQR, 5.0-23.0) months.

Optimal cut-off value to differentiate early versus late recurrence was determined by evaluating PRS at different time points after surgery using 2 -month intervals (Table 2). Of note, 8 months after surgery was associated with the largest difference in PRS among patients who developed early versus late recurrence (median PRS, early recurrence 27.0 vs. late recurrence 43.0 months, $p=$ 0.008 ) (Fig. 2a). In turn, 8 months was used as the optimal cut-off time for differentiating early versus late recurrence (Table 2). The cut-off time of 8 months was also strongly associated with early versus late intrahepaticonly recurrence (median PRS, early recurrence 27.0 vs. late recurrence 43.0 months, $p=0.019$ ) (Supplementary Table 1). Moreover, patients who recurred within 8 months after surgery had markedly worse OS versus patients who experienced recurrence beyond 8 months after surgery (median OS, early recurrence 32.0 versus late recurrence 74.0 months, $p<0.001$ ) (Fig. 2b). In particular, patients who recurred early had a 2 -fold higher risk of death versus patients who recurred later after adjustment for tumor characteristics, as well as treatment-related differences (HR, 2.2; 95\% CI, 1.3-3.6; $p=0.004$ ) (Supplementary Table 2).

Compared with late recurrence, early recurrence was more likely to be associated with worse tumor characteristics, such as high preoperative AFP (> $200 \mathrm{ng} / \mathrm{ml})$, large tumor size $(>5 \mathrm{~cm})$, advanced BCLC stage, poor tumor differentiation, as well as microvascular invasion (all $p<0.05$, Supplementary Table 3 ). These factors were also associated with a higher risk of intrahepatic-only recurrence among patients with early versus late recurrence (Supplementary Table 4). On multivariable analysis, increased AFP level (>200 ng/ml), large tumor size (> $5 \mathrm{~cm}$ ), R1 margin, microvascular invasion, and poor tumor differentiation were independent risk factors associated with early postoperative recurrence (all $p<0.05$, Table 4). In contrast, only tumor burden, including size and number, was independently correlated with late recurrence beyond 8 months after primary surgery (all $p<0.05$, Table 4).

\section{Recurrence Patterns and Treatment for Defined Early and Later Recurrence}

A majority of patients $(n=267,74.2 \%)$ who recurred had intrahepatic-only relapse, whereas 93 patients had extrahepatic-only metastasis $(n=64,17.8 \%)$ or extra- \pm intrahepatic recurrence $(n=29,8.1 \%)$. Of note, recurrence patterns and treatments were generally different among patients with early or late recurrence. Specifically, patients who recurred early within 8 months after primary surgery were more likely to recur extra- \pm intrahepatically versus patients who recurred beyond 8 months after initial resection $(35.5 \%$ vs. $19.8 \%, p=0.003)$. With regard to intrahepatic recurrence only, patients with early recurrence tended to have recurrent tumors that were larger in size and were less frequently amenable to secondary curatively treatment options compared with patients who experienced a late tumor recurrence (curative treatment percentage, $33.8 \%$ vs. $45.7 \%, p=0.080$ ) (Table 3).

Among 231 patients who had detailed information on treatment of intrahepatic-only recurrence, 96 (41.6\%) underwent curative resection/ablation, whereas 135 $(58.4 \%)$ received only palliative treatments (Table 4$)$. Of note, the OS after initial resection among patients undergoing curative treatments for intrahepatic-only recurrence was comparable to patients who had no recurrence during the study period (median OS, 139.0 vs. 140.0 months, $p=$ 0.561 ), yet better than patients treated with non- 
Table 1 Baseline demographics and clinicopathological variables of patients with versus without recurrence

\begin{tabular}{|c|c|c|c|c|}
\hline Variables & Overall $(n=1004)$ & No recurrence $(n=561)$ & Recurrence $(n=443)$ & $P$ \\
\hline Age, years & & & & 0.830 \\
\hline$\leq 60$ & $454(45.2 \%)$ & $252(44.9 \%)$ & $202(45.6 \%)$ & \\
\hline$>60$ & $550(54.8 \%)$ & $309(55.1 \%)$ & $241(54.4 \%)$ & \\
\hline Gender & & & & 0.495 \\
\hline Male & $788(78.6 \%)$ & $436(77.9 \%)$ & $352(79.6 \%)$ & \\
\hline Female & $214(21.4 \%)$ & $214(22.1 \%)$ & $90(20.4 \%)$ & \\
\hline Diabetes mellitus & $259(26.3 \%)$ & $146(26.4 \%)$ & $113(26.3 \%)$ & 0.979 \\
\hline Chronic alcohol intake & $186(18.8 \%)$ & $87(19.9 \%)$ & $99(17.9 \%)$ & 0.432 \\
\hline HBV infection & $227(25.8 \%)$ & $130(26.4 \%)$ & $97(25.1 \%)$ & 0.648 \\
\hline HCV infection & $212(21.3 \%)$ & $109(19.5 \%)$ & $103(23.6 \%)$ & 0.119 \\
\hline $\mathrm{AFP}, \mathrm{ng} / \mathrm{ml}$ & & & & $<0.001$ \\
\hline$\leq 400$ & $655(79.4 \%)$ & $378(84.8 \%)$ & $277(73.1 \%)$ & \\
\hline$>400$ & $170(20.6 \%)$ & $68(15.2 \%)$ & $102(26.9 \%)$ & \\
\hline Child-Pugh classification & & & & 0.526 \\
\hline A & $716(93.8 \%)$ & $408(93.4 \%)$ & $308(94.5 \%)$ & \\
\hline B & $47(6.2 \%)$ & $29(6.6 \%)$ & $18(5.5 \%)$ & \\
\hline Surgery types & & & & 0.008 \\
\hline Minimally invasive & $275(27.5 \%)$ & $172(30.9 \%)$ & $103(23.3 \%)$ & \\
\hline Open & $724(72.5 \%)$ & $385(69.1 \%)$ & $339(76.7 \%)$ & \\
\hline Extent of resection & & & & 0.082 \\
\hline Minor & $676(69.9 \%)$ & $394(72.7 \%)$ & $282(67.0 \%)$ & \\
\hline Major & $291(30.1 \%)$ & $152(27.8 \%)$ & $139(33.0 \%)$ & \\
\hline Type of resection & & & & 0.379 \\
\hline Anatomic & $799(82.6 \%)$ & $446(81.7 \%)$ & $353(83.8 \%)$ & \\
\hline Non-anatomic & $168(17.4 \%)$ & $100(18.3 \%)$ & $68(16.2 \%)$ & \\
\hline Maximum tumor size, $\mathrm{cm}$ & & & & $<0.001$ \\
\hline$\leq 5$ & $524(52.2 \%)$ & $323(57.6 \%)$ & $201(45.4 \%)$ & \\
\hline$>5$ & $480(47.8 \%)$ & $238(42.4 \%)$ & $242(54.6 \%)$ & \\
\hline Tumor number & & & & $<0.001$ \\
\hline Single & $885(88.1 \%)$ & $513(91.4 \%)$ & $372(84.0 \%)$ & \\
\hline Multiple & $119(11.9 \%)$ & $48(8.6 \%)$ & $71(16.0 \%)$ & \\
\hline Tumor location & & & & 0.018 \\
\hline Unilobar & $877(92.2 \%)$ & $504(94.0 \%)$ & $373(89.9 \%)$ & \\
\hline Bilobar & $74(7.8 \%)$ & $32(6.0 \%)$ & $42(10.1 \%)$ & \\
\hline BCLC staging & & & & 0.002 \\
\hline $0 / \mathrm{A}$ & $800(84.9 \%)$ & $468(88.1 \%)$ & $332(80.8 \%)$ & \\
\hline $\mathrm{B} / \mathrm{C}$ & $142(15.1 \%)$ & $63(11.9 \%)$ & $79(19.2 \%)$ & \\
\hline Liver cirrhosis & $439(43.8 \%)$ & $254(45.4 \%)$ & $185(41.9 \%)$ & 0.267 \\
\hline Grade & & & & $<0.001$ \\
\hline Well to moderate & $741(77.4 \%)$ & $434(81.7 \%)$ & $307(72.1 \%)$ & \\
\hline Poor & $216(22.6 \%)$ & $97(18.3 \%)$ & $119(27.9 \%)$ & \\
\hline Microvascular invasion & $284(32.5 \%)$ & $144(28.9 \%)$ & $140(37.4 \%)$ & 0.007 \\
\hline Capsule involvement & $280(36.6 \%)$ & $158(37.0 \%)$ & $122(36.1 \%)$ & 0.796 \\
\hline Margin status & & & & 0.689 \\
\hline R0 & $902(90.0 \%)$ & $506(90.4 \%)$ & $396(89.6 \%)$ & \\
\hline $\mathrm{R} 1$ & $100(10.0 \%)$ & $54(9.6 \%)$ & $46(10.4 \%)$ & \\
\hline Postoperative complications & $411(41.4 \%)$ & $222(40.1 \%)$ & $189(43.0 \%)$ & 0.372 \\
\hline Severe complications (III-IV) & $87(8.8 \%)$ & $49(8.9 \%)$ & $38(8.6 \%)$ & 0.901 \\
\hline
\end{tabular}

$P$ values that are significant are in italics

$B M I$ body mass index, ASA American Society of Anesthesiologists, NASH non-alcoholic steatohepatitis, PSC primary sclerosing cholangitis, PLT platelet, $A L T$ alanine aminotransferase, $A S T$ aspartate aminotransferase, $A F P \alpha$-fetoprotein, $B C L C$ Barcelona Clinic Liver Cancer

curativepalliative options for the intrahepatic recurrence (median OS, 139.0 vs. 43.0 months, $p<0.001$ ) (Fig. 3a). Interestingly, when stratified by recurrence time (early versus late recurrence), patients who developed early intrahepatic recurrence still had a worse OS even after curative treatment versus patients who never recurred (median OS, 69.0 vs. 140.0 months, $p=0.036$ ), but OS was better than patients who received palliative treatments for early recurrence (median OS, 69.0 vs. 21.0 months, $p<0.001$ ) (Fig. 3b). In contrast, patients with late recurrence had a comparable OS after curative treatment versus patients who had no recurrence (median OS, 139.0 vs. 140.0 months, $p=0.738$ ) (Fig. 3c). Multivariable analysis demonstrated that curative versus non-curative treatments 


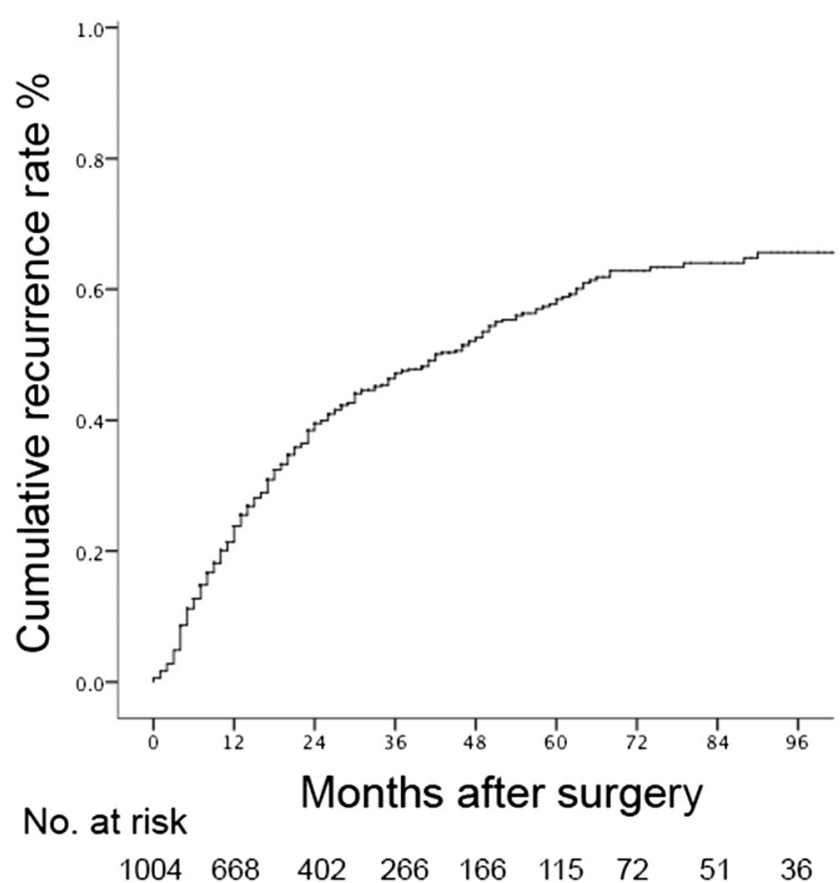

Fig. 1 Cumulative incidence of recurrence for $\mathrm{HCC}$ after curative resection

of recurrence (HR 0.5, 95\% CI 0.3-0.9) was independently associated with a favorable PRS (Supplementary Table 2).

\section{Discussion}

Recurrence is common among patients with $\mathrm{HCC}$ after curative resection. $\left[{ }^{3,7,13-17}\right]$ Data on timing of HCC recurrence, as well as an evidence-based definition of early versus late recurrence after curative resection, remain poorly defined. While several investigators have proposed 24 months as a cut-off value to differentiate early versus late recurrence, $[3$, ${ }^{7}$ ] other studies adopted an earlier time point of 6 or 12 months as the cut-off. $\left[{ }^{14,16}\right]$ These past studies suffered from some mythological weakness in that the proposed cut-off values were not established based on a robust statistical assessment of relevant clinical parameters. The current study was important because we specifically examined multiple short-term intervals (i.e., 2 months) to establish 8 months as the recurrencefree interval to define early versus late recurrence relative to PRS. Specifically, patients who developed early recurrence within 8 months had markedly worse PRS, as well as OS versus patients who recurred beyond 8 months after resection of HCC. Of note, the characteristics of the initial HCC, as well as recurrence patterns and post-recurrence treatments, were different among patients who developed early versus late recurrence. Specifically, patients who recurred within 8 months were more likely to recur at an extra- \pm intrahepatic site versus patients who recurred beyond 8 months after the initial HCC resection ( $35.5 \%$ vs. $19.8 \%, p=0.003)$. Of note, patients who developed a late recurrence had comparable OS after curativeintent re-treatment of recurrence versus patients who had no recurrence (median OS, 139.0 vs. 140.0 months, $p=0.738$ ). In contrast, patients who developed early intrahepatic recurrence still had a worse OS even after curative re-treatments compared with patients who did never recur (median OS, 69.0 vs. 140.0 months, $p=0.036$ ).

Survival after recurrence likely reflects the malignant characteristics of recurrent tumor and therefore data on early versus late recurrence may be important for prognostic purposes.
Table 2 Defining cut-off thresholds of early versus late recurrence using PRS

\begin{tabular}{|c|c|c|c|c|c|}
\hline \multirow{2}{*}{$\begin{array}{l}\text { Cut-off (months after } \\
\text { surgery) }\end{array}$} & \multicolumn{2}{|c|}{ Early-recurrence cohort } & \multicolumn{2}{|c|}{ Late-recurrence cohort } & \multirow{2}{*}{$\begin{array}{l}P \text { value for } \\
\text { PRS }\end{array}$} \\
\hline & $\begin{array}{l}\text { Median PRS } \\
\text { (month) }\end{array}$ & $\begin{array}{l}\text { Median OS } \\
\text { (month) }\end{array}$ & $\begin{array}{l}\text { Median PRS } \\
\text { (month) }\end{array}$ & $\begin{array}{l}\text { Median OS } \\
\text { (month) }\end{array}$ & \\
\hline 2 & 41.0 & 42.0 & 36.0 & 65.0 & 0.711 \\
\hline 4 & 44.0 & 44.0 & 35.0 & 68.0 & 0.499 \\
\hline 6 & 31.0 & 35.0 & 41.0 & 71.0 & 0.028 \\
\hline 8 & 27.0 & 32.0 & 43.0 & 74.0 & 0.008 \\
\hline 10 & 27.0 & 32.0 & 43.0 & 74.0 & 0.033 \\
\hline 12 & 31.0 & 36.0 & 43.0 & 75.0 & 0.054 \\
\hline 14 & 31.0 & 42.0 & 43.0 & 80.0 & 0.107 \\
\hline 16 & 31.0 & 41.0 & 45.0 & 84.0 & 0.051 \\
\hline 18 & 31.0 & 41.0 & 45.0 & 87.0 & 0.043 \\
\hline 20 & 35.0 & 42.0 & 45.0 & 87.0 & 0.094 \\
\hline 22 & 35.0 & 44.0 & 40.0 & 87.0 & 0.299 \\
\hline 24 & 41.0 & 50.0 & 40.0 & 118.0 & 0.409 \\
\hline
\end{tabular}

$P$ values that are significant are in italics

$P R S$ post-recurrence survival, $O S$ overall survival 
a

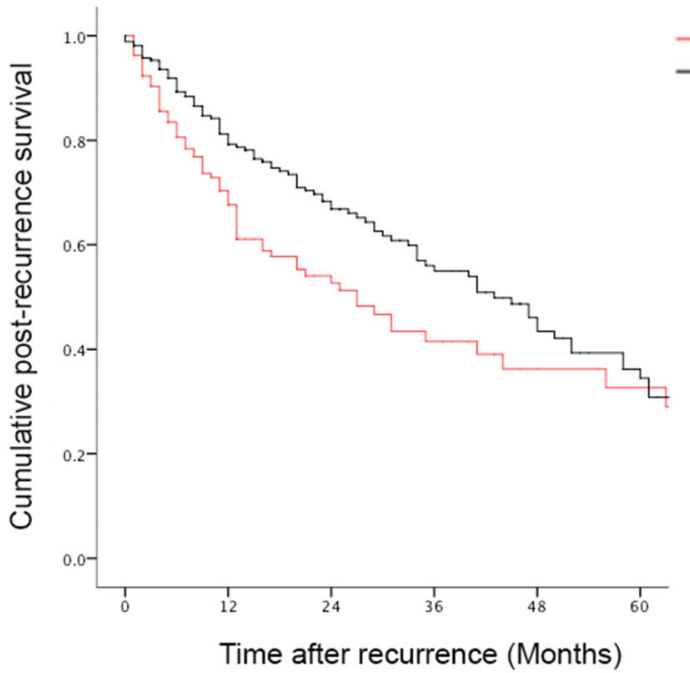

No. at risk b

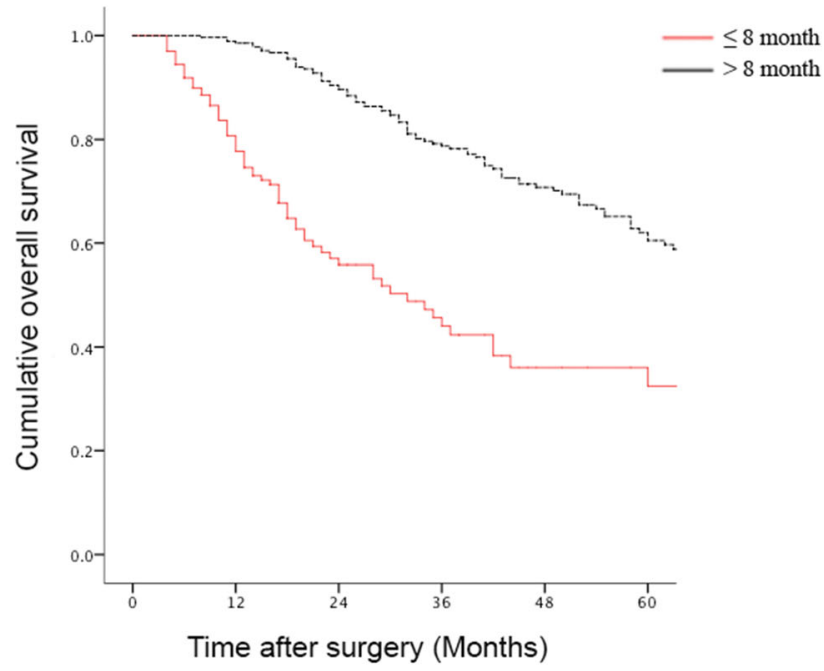

No. at risk

$\begin{array}{lllllll}- & 165 & 107 & 48 & 28 & 13 & 10 \\ -278 & 270 & 227 & 159 & 108 & 79\end{array}$

Fig. 2 Post-recurrence survival (a) and overall survival (b) among patients with early versus late recurrence

Indeed, PRS has previously been adopted to differentiate early versus late recurrence among patients with pancreatic ductal adenocarcinoma. $\left[{ }^{11}\right]$ In the current study, 8 months was utilized as the optimal cut-off to differentiate early versus late recurrence, as patients who recurred within 8 months after surgery had markedly worse PRS versus patients who recurred beyond 8 months. Recently, Xing et al. had proposed a similar cut-off of 8 months to define early recurrence in a large series of patients from China. $\left[{ }^{17}\right]$ The current study builds on this previous work in that the data externally validated the 8-month cut-off using a large multi-center international cohort of patients from mostly Western centers.
Interestingly, median PRS among patients with an early versus late recurrence was 8.4 and 21.3 months, respectively, in the study by Xing et al. In contrast, PRS was markedly better among patients with early (27.0 months) or late (43.0 months) in the current study. $\left[{ }^{17}\right]$ The reason for the disparate results was undoubtedly multifactorial and may have been related to the predominance of HBV-related HCC (90.6\%) and more advanced tumor stage in the exclusively East Asian cohort in the study by Xing et al. $\left[{ }^{17}\right]$ To this point, the current multi-institutional international cohort was mostly composed of patients with Non-B Non-C HCC (52.9\%) patients. Of note, despite the differences in the baseline characteristics in

Table 3 Recurrence patterns of patients with early versus late recurrence

\begin{tabular}{llll}
\hline Variables & Total $(n=443)$ & Early recurrence $(n=165)$ & Late recurrence $(n=278)$ \\
\hline $\begin{array}{l}\text { Local recurrence } \\
\text { Recurrence site }\end{array}$ & $38(10.6 \%)$ & $15(10.9 \%)$ & $23(10.4 \%)$ \\
$\quad$ Intrahepatic & $267(74.2 \%)$ & $89(64.5 \%)$ & $178(80.2 \%)$ \\
Extrahepatic & $64(17.8 \%)$ & $32(23.2 \%)$ & $32(14.4 \%)$ \\
Both & $29(8.1 \%)$ & $17(12.3 \%)$ & $12(5.4 \%)$ \\
Intrahepatic-only recurrence & & & 0.872 \\
Number of recurrent lesions & & & \\
Single & $146(60.6 \%)$ & $47(58.8 \%)$ & $99(61.5 \%)$ \\
$\quad$ Multiple & $95(39.4 \%)$ & $33(41.3 \%)$ & $62(38.5 \%)$ \\
Recurrent tumor size & $2.2 \pm 2.3$ & $2.5 \pm 2.3$ & $2.1 \pm 2.3$ \\
Treatment for recurrence & & & 6.682 \\
Curative & $96(41.6 \%)$ & $27(33.8 \%)$ & $82(54.3 \%)$ \\
Non-curative & $135(58.4 \%)$ & $53(66.3 \%)$ & 0.078 \\
\hline
\end{tabular}

$P$ values that are significant are in italics

${ }^{\text {a }}$ Curative: resection or ablation; non-curative: chemotherapy, TACE or no treatment 
Table 4 Multivariable logistic regression analysis of risk factors for early and late recurrence

\begin{tabular}{|c|c|c|c|c|}
\hline \multirow[t]{2}{*}{ Variables } & \multicolumn{2}{|l|}{ Univariate } & \multicolumn{2}{|l|}{ Multivariable } \\
\hline & OR $(95 \% \mathrm{CI})$ & $P$ & OR $(95 \% \mathrm{CI})$ & $P$ \\
\hline \multicolumn{5}{|l|}{ Early recurrence } \\
\hline Age (years), $>60$ vs. $\leq 60$ & $1.0(0.7-1.4)$ & 0.986 & & \\
\hline $\operatorname{AFP}(\mathrm{ng} / \mathrm{ml}),>200$ vs. $\leq 200$ & $3.5(2.4-5.2)$ & $<0.001$ & $2.8(1.8-4.4)$ & $<0.001$ \\
\hline Child-Pugh grade, B vs. A & $0.7(0.3-1.7)$ & 0.409 & & \\
\hline Tumor number, multiple vs. single & $1.9(1.2-3.0)$ & 0.007 & $1.3(0.7-2.2)$ & 0.439 \\
\hline Tumor size, $>5$ vs. $\leq 5$ & $2.0(1.4-2.8)$ & $<0.001$ & $1.6(1.0-2.6)$ & 0.031 \\
\hline Cirrhosis, yes vs. no & $1.2(0.8-1.6)$ & 0.417 & & \\
\hline Macrovascular invasion, yes vs. no & $3.2(1.8-5.9)$ & $<0.001$ & $1.2(0.6-2.7)$ & 0.572 \\
\hline Resection margin, R1 vs. R0 & $1.9(1.2-3.1)$ & 0.008 & $2.1(1.1-3.8)$ & 0.022 \\
\hline Liver capsule involvement, yes vs. no & $1.0(0.7-1.5)$ & 0.841 & & \\
\hline Microvascular invasion, yes vs. no & $3.2(2.2-4.7)$ & $<0.001$ & $1.9(1.2-3.0)$ & 0.004 \\
\hline Tumor grade, poor vs. well/moderate & $2.8(1.9-4.1)$ & $<0.001$ & $2.0(1.3-3.1)$ & 0.002 \\
\hline \multicolumn{5}{|l|}{ Late recurrence } \\
\hline Age (years), $>60$ vs. $\leq 60$ & $1.0(0.7-1.4)$ & 0.921 & & \\
\hline $\operatorname{AFP}(\mathrm{ng} / \mathrm{ml}),>200$ vs. $\leq 200$ & $1.1(0.8-1.8)$ & 0.496 & & \\
\hline Child-Pugh grade, B vs. A & $0.9(0.5-1.9)$ & 0.880 & & \\
\hline Tumor number, multiple vs. single & $1.7(1.1-2.6)$ & 0.027 & $1.6(1.0-2.5)$ & 0.035 \\
\hline Tumor size, $>5$ vs. $\leq 5$ & $1.4(1.0-1.8)$ & 0.031 & $1.4(1.0-1.8)$ & 0.040 \\
\hline Macrovascular invasion, yes vs. no & $2.0(0.9-4.6)$ & 0.114 & & \\
\hline Cirrhosis, yes vs. no & $1.1(0.8-1.5)$ & 0.470 & & \\
\hline Resection margin, R1 vs. R0 & $0.7(0.4-1.1)$ & 0.144 & & \\
\hline Liver capsule involvement, yes vs. no & $0.9(0.7-1.3)$ & 0.657 & & \\
\hline Microvascular invasion, yes vs. no & $0.9(0.6-1.3)$ & 0.544 & & \\
\hline Tumor grade, poor vs. well/moderate & $1.1(0.8-1.6)$ & 0.544 & & \\
\hline
\end{tabular}

$P$ values that are significant are in italics

$O S$ overall survival, $A L T$ alanine aminotransferase, $A S T$ aspartate aminotransferase, $A F P \alpha$-fetoprotein, $B C L C$ Barcelona Clinic Liver Cancer, $R F S$ recurrence-free survival

the two cohorts, the data in both studies demonstrated 8 months as the optimal cut-off to differentiate early versus late recurrence.

Timing of recurrence likely indicates distinct relapse mechanisms and different prognoses. For example, early recurrence is likely caused by dissemination of primary tumor and occult metastasis before surgical resection. Thus, early-recurring tumors have generally been more associated with aggressive characteristics and worse outcomes. $\left[{ }^{15,18}\right]$ Risk stratification to identify patients most at risk for early recurrence is clinically important in the postoperative setting to plan screening and surveillance for recurrence. $\left[{ }^{5}\right]$ In addition, several a

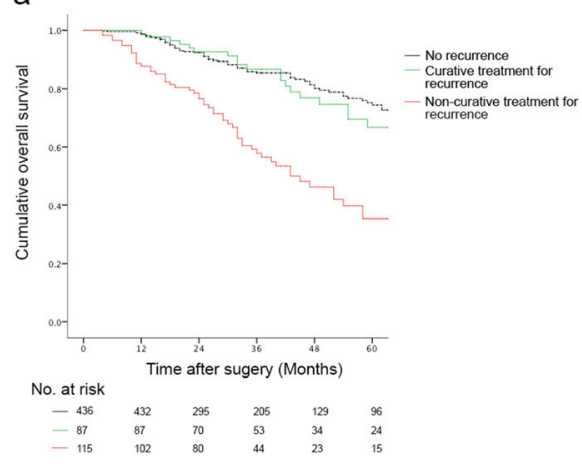

b

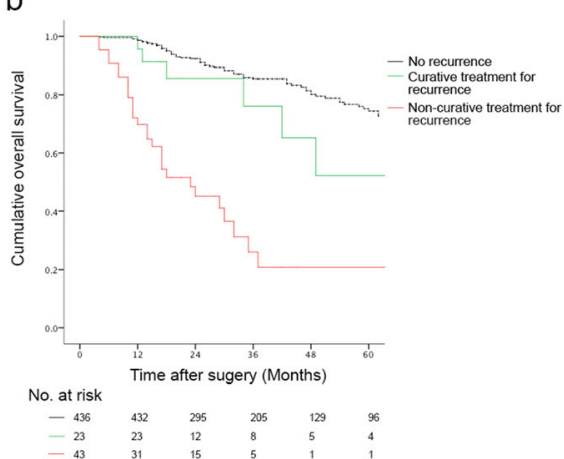

C

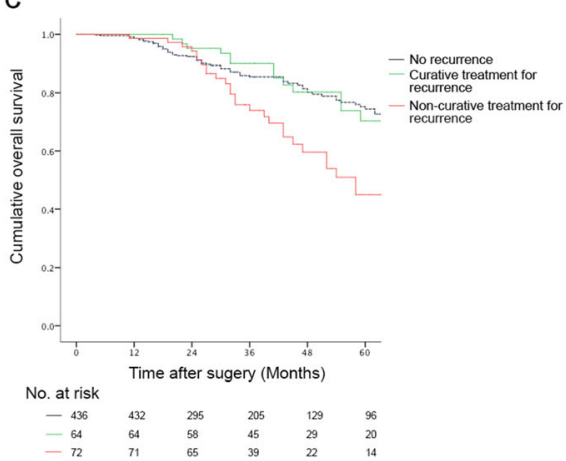

Fig. 3 Overall survival according to treatment of recurrence for all patients (a) and the subgroup of patients with early (b) or late (c) recurrence 
investigators have even suggested that patients at risk of early HCC recurrence may benefit from adjuvant treatments, including intra-arterial therapy, as well as be screened for enrollment in clinical trials given that there is no established standard adjuvant care for high-risk patients. $\left[{ }^{19}\right]$ To this point, we identified several tumor-specific factors including high AFP level, large tumor size, microvascular invasion and poor differentiation, as well as narrow surgical margins $(<1 \mathrm{~mm})$ that were independently associated with risk of early recurrence within 8 months after initial surgery. In addition, early recurrence was more commonly noted to be at an extra-hepatic site versus late recurrence. Collectively, the data suggest that patients with these features should be screened frequently in the immediate post-operative period with a particular focus on surveillance of extra-hepatic disease.

Data from the current study also supported the potential benefit of re-treatment for recurrence relative to long-term survival among patients with intrahepatic-only recurrence. $\left[{ }^{18,20,21}\right]$ A previous meta-analysis had reported median 5year OS after repeat resection, ablation, or transarterial chemoembolization for recurrent HCC of $35.2 \%$ (22-84\%) and $48.3 \%(24-83 \%), 15.5 \%(0-56 \%)$, respectively. $\left[{ }^{2}\right]$ These data were consistent with data in the current study, which noted a 5 -year OS of $66.0 \%$ after repeat resection/ ablation versus only $37.0 \%$ after palliative treatment. Of note, patients who underwent curative re-treatment of late recurrence had an even better long-term prognosis, which was even comparable to OS of patients who had no recurrence (median OS, 139.0 vs. 140.0 months). While OS following curativeintent treatment of early recurrence was inferior to outcomes among patients with no recurrence (median OS, 69.0 vs. 140.0 months, $p=0.036$ ), prognosis was still markedly better than patients who received palliative only treatments for early recurrence (median OS, 69.0 vs. 21.0 months, $p<0.001$ ). While possible clinical differences and selection bias make definitive conclusions not possible, the data did suggest that repeat curative-intent surgery for well selected patients with HCC recurrence may be warranted and provide a benefit.

The current study demonstrated that several clinicopathologic factors were strongly associated with increased risk of early recurrence. Identifying patients at high risk for early relapse is of clinical significance. Specifically, these patients may be appropriate to be enrolled in clinical trials to receive neoadjuvant and/or adjuvant treatments. In addition, these findings also suggest that perhaps a subset of patients at very high risk of early recurrence may be better served with liverdirected therapies other than surgery. Moreover, implementing more stringent surveillance of disease within 8 months after surgical resection in the subgroup of patients at high risk of early recurrence may help in detection of early recurrence and utilization of secondary curative treatments.

The present study should be interpreted in light of several limitations. While the international multi-institutional-based cohort increased sample size and generalizability, patient selection, surgical procedures, as well as follow-up strategies may have varied at the different centers. Detailed information about the systemic and intra-arterial therapy was not available, and therefore was not considered. In addition, some patients with recurrence received more than one treatment modality for the recurrence. The current study considered the initial treatment of the recurrence and based OS calculations from the time of the first treatment of any recurrence.

In conclusion, a large number of patients with HCC recurred following curative-intent resection of HCC. Using a large multi-center international cohort of patients, we identified 8 months as the cut-off value to differentiate early versus late recurrence, which was consistent with data from a previous study based exclusively on Chinese patients. $\left[{ }^{17}\right]$ Patients who experienced an early recurrence within 8 months following resection had a markedly shortened PRS versus patients who recurred beyond 8 months after initial resection. Curative-intent treatment for recurrent intrahepatic tumors was associated with reasonable long-term outcomes and therefore should be considered for a select group of patients.

Funding Information Tao Wei and Xu-Feng Zhang were supported by the Clinical Research Award of the First Affiliated Hospital of Xi'an Jiaotong University of China (No. XJTU1AF-CRF-2017-004).

\section{Compliance with Ethical Standards}

Conflict of Interest The authors declare that they have no conflict of interest.

\section{References}

1. Villanueva A. Hepatocellular Carcinoma. N Engl J Med. 2019;380: 1450-1462.

2. Erridge S, Pucher PH, Markar SR, et al. Meta-analysis of determinants of survival following treatment of recurrent hepatocellular carcinoma. Br J Surg. 2017;104:1433-1442.

3. Portolani N, Coniglio A, Ghidoni S, et al. Early and late recurrence after liver resection for hepatocellular carcinoma: prognostic and therapeutic implications. Ann Surg. 2006;243:229-235.

4. Tabrizian P, Jibara G, Shrager B, Schwartz M, Roayaie S. Recurrence of hepatocellular cancer after resection: patterns, treatments, and prognosis. Ann Surg. 2015;261:947-955.

5. Chan AWH, Zhong J, Berhane S, et al. Development of pre and post-operative models to predict early recurrence of hepatocellular carcinoma after surgical resection. J Hepatol. 2018;69:1284-1293.

6. Xu XF, Xing H, Han J, et al. Risk factors, patterns, and outcomes of late recurrence after liver resection for hepatocellular carcinoma: a multicenter study From China. JAMA Surg. 2019;154:209-217.

7. Imamura $\mathrm{H}$, Matsuyama $\mathrm{Y}$, Tanaka $\mathrm{E}$, et al. Risk factors contributing to early and late phase intrahepatic recurrence of hepatocellular carcinoma after hepatectomy. J Hepatol. 2003;38:200-207.

8. Ding X, He M, Chan AWH, et al. Genomic and epigenomic features of primary and recurrent hepatocellular carcinomas. Gastroenterology. 2019;157:1630-1645.e1636. 
9. Shah SA, Cleary SP, Wei AC, et al. Recurrence after liver resection for hepatocellular carcinoma: risk factors, treatment, and outcomes. Surgery. 2007;141:330-339.

10. He W, Peng B, Tang Y, et al. Nomogram to predict survival of patients with recurrence of hepatocellular carcinoma after surgery. Clin Gastroenterol Hepatol. 2018;16:756-764.e710.

11. Groot VP, Gemenetzis G, Blair AB, et al. Defining and predicting early recurrence in 957 patients with resected pancreatic ductal adenocarcinoma. Ann Surg. 2019;269:1154-1162.

12. Slankamenac K, Graf R, Barkun J, Puhan MA, Clavien PA. The comprehensive complication index: a novel continuous scale to measure surgical morbidity. Ann Surg. 2013;258:1-7.

13. Cheng Z, Yang P, Qu S, et al. Risk factors and management for early and late intrahepatic recurrence of solitary hepatocellular carcinoma after curative resection. HPB (Oxford). 2015;17:422-427.

14. Lu X, Zhao H, Yang H, et al. A prospective clinical study on early recurrence of hepatocellular carcinoma after hepatectomy. J Surg Oncol. 2009;100:488-493.

15. Poon RT, Fan ST, Ng IO, Lo CM, Liu CL, Wong J. Different risk factors and prognosis for early and late intrahepatic recurrence after resection of hepatocellular carcinoma. Cancer. 2000;89:500-507.

16. Shah SA, Greig PD, Gallinger S, et al. Factors associated with early recurrence after resection for hepatocellular carcinoma and outcomes. J Am Coll Surg. 2006;202:275-283.
17. Xing H, Zhang WG, Cescon M, et al. Defining and predicting early recurrence after liver resection of hepatocellular carcinoma: a multiinstitutional study. HPB (Oxford). 2019.

18. Yoh T, Seo S, Taura K, et al. Surgery for recurrent hepatocellular carcinoma: achieving long-term survival. Ann Surg. 2019.

19. Bruix J, Takayama T, Mazzaferro V, et al. Adjuvant sorafenib for hepatocellular carcinoma after resection or ablation (STORM): a phase 3, randomised, double-blind, placebo-controlled trial. Lancet Oncol. 2015;16:1344-1354.

20. Huang ZY, Liang BY, Xiong M, et al. Long-term outcomes of repeat hepatic resection in patients with recurrent hepatocellular carcinoma and analysis of recurrent types and their prognosis: a single-center experience in China. Ann Surg Oncol. 2012;19: 2515-2525.

21. Minagawa M, Makuuchi M, Takayama T, Kokudo N. Selection criteria for repeat hepatectomy in patients with recurrent hepatocellular carcinoma. Ann Surg. 2003;238:703-710.

Publisher's Note Springer Nature remains neutral with regard to jurisdictional claims in published maps and institutional affiliations.

\section{Affiliations}

Tao Wei $^{1,2} \cdot$ Xu-Feng Zhang $^{1} \cdot$ Fabio Bagante $^{3,4} \cdot$ Francesca Ratti $^{5} \cdot$ Hugo P. Marques $^{6} \cdot$ Silvia Silva $^{6}$.

Olivier Soubrane ${ }^{7} \cdot$ Vincent Lam $^{8} \cdot$ George A. Poultsides $^{9} \cdot$ Irinel Popescu ${ }^{10} \cdot$ Razvan Grigorie $^{10} \cdot$ Sorin Alexandrescu $^{10}$. Guillaume Martel $^{11} \cdot$ Aklile Workneh $^{11} \cdot$ Alfredo Guglielmi $^{4} \cdot$ Tom Hugh $^{12} \cdot$ Yi Lv $^{1} \cdot$ Luca Aldrighetti $^{5}$. Timothy M. Pawlik ${ }^{3}$ (D)

1 Department of Hepatobiliary Surgery and Institute of Advanced Surgical Technology and Engineering, The First Affiliated Hospital of Xi'an Jiaotong University, Xi'an, China

2 Department of Hepatobiliary and Pancreatic Surgery, The First Affiliated Hospital, Zhejiang University School of Medicine, Hangzhou, China

3 Department of Surgery, Division of Surgical Oncology, The Ohio State University Wexner, Medical Center and James Comprehensive Cancer Center, Columbus, OH, USA

4 Department of Surgery, University of Verona, Verona, Italy

5 Department of Surgery, Ospedale San Raffaele, Milan, Italy
6 Department of Surgery, Curry Cabral Hospital, Lisbon, Portugal

7 Department of Hepatobiliopancreatic Surgery, APHP, Beaujon Hospital, Clichy, France

8 Department of Surgery, Westmead Hospital, Sydney, Australia

9 Department of Surgery, Stanford University, Stanford, CA, USA

10 Department of Surgery, Fundeni Clinical Institute, Bucharest, Romania

11 Department of Surgery, University of Ottawa, Ottawa, Canada

12 Department of Surgery, The University of Sydney, School of Medicine, Sydney, Australia 